RESEARCH NOTE

\section{IgE and IgG4 Antibodies in Subjects Reinfected with Schistosoma mansoni in an Endemic Area of Northeast Brazil}

\section{Yara M Gomes ${ }^{+}$, Felipe C Gomes, Veridiana C Furtado, Kirte M Teixeira, Wayner V Souza, Frederico GC Abath}

Departamento de Imunologia, Centro de Pesquisas Aggeu Magalhães-Fiocruz, Av. Moraes Rego s/no Cidade Universitária, 50670-420 Recife, PE, Brasil

Key words: schistosomiasis - IgE and IgG4 antibodies - Schistosoma mansoni

Analysis of the immune response of resistant and susceptible subjects in endemic areas indicated that resistance was associated with enhanced antiparasite-IgE levels and that reinfection occurred when patients were producing high levels of antibodies that could compete with IgE (AE Butterworth et al. 1985 Trans R Soc Trop Med Hyg 79: 393-408, P Hagan et al. 1987 Trans $R$ Soc Trop Med Hyg 81: 938-946, P Rihet et al. 1991 Eur J Immunol 21: 2679-2686, DW Dunne et al. 1992 Eur J Immunol 22: 1483-1494). The results of these reinfection studies suggest that acquired immunity develops slowly with age and that, although other factors can not be excluded, IgE specific antibodies play an important role in anti-schistosome resistance.

Recently, the role of different factors involved in Schistosoma mansoni infection (including the nutritional status of the population) was evaluated by E Coutinho et al. (1997 Mem Inst Oswaldo Cruz. 92: 710-715) in a population living in two contiguous endemic villages (Itapinassu and São Joaquim), in northeast Brazil. The patients were

This work was partially funded by Facepe, WHO and CNPq.

${ }^{+}$Corresponding author. Fax: +55-81-453.1911. E-mail: yara@cpqam.fiocruz.br

Received 4 May 1998

Accepted 31 August 1998 identified by stool examinations (WA Hoffman et al. 1934 Puerto Rico J Publ Hlth Trop Med 9: 626653, N Katz et al. 1972 Rev Inst Med Trop São Paulo 14: 397-400) and the intensity of infection was classified as light $(<100 \mathrm{epg})$, moderate (101$400)$ and severe (>400 epg). All patients positive for $S$. mansoni were treated with oxaminiquine in a single dose $(15 \mathrm{mg} / \mathrm{kg}$ for adults and $20 \mathrm{mg} / \mathrm{kg}$ for patients under 15 years old). A previous therapy against other helminth infections was carried out with mebendazole and/or thiabendazole (Coutinho et al. loc. cit.) before starting the study.

In the present communication we evaluated the influence of the IgE and IgG4 levels on the resistance and susceptibility to infection by $S$. mansoni of 141 patients living in the area mentioned above.

Blood was obtained by venipuncture, six months after treatment, and serum was stored at $-20^{\circ} \mathrm{C}$ until use. Soluble worm antigen preparation (SWAP) and soluble egg antigen (SEA) were prepared using stantard procedures (DG Colley et al. 1977 Int Arch Allergy Appl Immunol 53: 420-443, G Gazzinelli et al. 1983 J Immunol 130: 28912895). Antigen preparations were dialysed against destilled water and the protein concentration determined by the method of OH Lowry et al. (1951 J Biol Chem 193: 263-275). IgE and IgG4 specific antibody response to SWAP and SEA was evaluated by ELISA (P Hagan 1991 Nature 349: 243245). Each antigen was diluted in $0.05 \mathrm{M} \mathrm{Na}_{2} \mathrm{CO}_{3}$ buffer, $\mathrm{pH}$ 9.6, onto flat-bottomed microtitre plates at the optimum concentration determined by chequerboard titration using pooled positive and negative control sera. The levels of specific IgE and IgG4 bound by these antigens was determined using mouse monoclonal anti-human IgE and IgG4 ( $\mathrm{Fc}$ fragments). Assays were developed using a horseradish peroxidase-conjugated rabbit antimouse IgG. Optimum concentrations of all reagents were determined by titration. Statistical analysis was performed using the software EPIINFO V 6.03. Analysis of variance was employed to determine the diferences between frequences; $p<0.05$ was considered to be statistically significant.

In the present note only the results concerning reinfection after treatment are mentioned. Reinfection after treatment was moderate and severe in young adults while in subjects older than 35 years, the intensities were lighter (data not shown). This kind of age-related intensity of infection is a typical epidemiological feature of schistosomiasis. An association between age and levels of IgE schistosome-specific antibodies was found. IgE levels to SWAP antigen were maximal in the $35+$ age group $(\mathrm{p}<0.05)$ and the age-IgE profile follows that expected for an antibody involved in resistance to infection. The IgE levels to SEA antigen was also 
slightly more elevated in the 35+ age group although this was not significant (Fig. 1). Similar results were found by Hagan (1991 loc. cit.). The stratified analysis of the IgG4 levels to SWAP and SEA was not well defined and conclusive. IgG4 levels were more elevated to SEA and SWAP in the young adults and in the $35+$ age group although this was not statistically significant (Fig. 2). Taken into account the pattern of age related intensity of

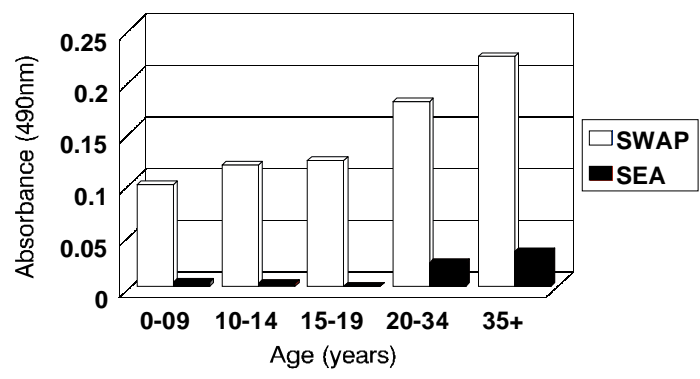

Fig. 1: IgE specific antibody responses to soluble worm antigen preparation (SWAP) and soluble egg antigens (SEA) of Schistosoma mansoni six months after treatment in patients living in an endemic area. infection (high in young adults, low in adults), the present results suggest that the levels of IgE antibodies to SWAP may be used as a marker of resistance against schistosomiasis, supporting results from other groups (Hagan 1991 loc. cit., DW Dunne et al. 1997 Parasite Immunol 19: 79-89).

Acknowledgements: to Mr Mineo Nakazawa, $\mathrm{Mr}$ Rafael Guimarães, and Mrs Rosineide Lira for technical assistance.

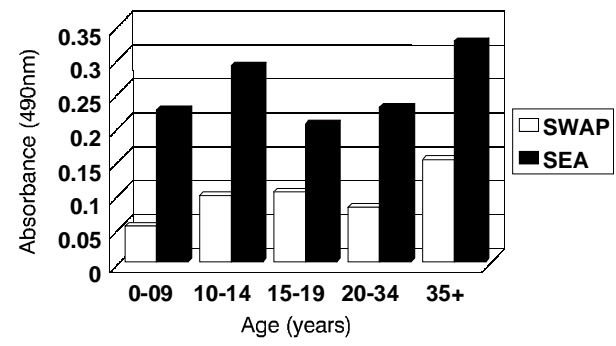

Fig. 2: IgG4 antibody responses to soluble worm antigen preparation (SWAP) and soluble egg antigens (SEA) of Schistosoma mansoni six months after treatment in patients living in an endemic area. 